\title{
Photonic clusters formed by dielectric microspheres: Numerical simulations
}

\author{
Jack Ng, Z. F. Lin,* C. T. Chan, ${ }^{\dagger}$ and Ping Sheng \\ Physics Department, Hong Kong University of Science and Technology, Clear Water Bay, Hong Kong \\ (Received 28 February 2005; revised manuscript received 31 May 2005; published 24 August 2005)
}

\begin{abstract}
We show through rigorous calculations that small dielectric microspheres can be organized by an incident electromagnetic plane wave into stable geometric configurations, which we call photonic clusters. The longranged optical binding force arises from the multiple scattering between the spheres. A photonic cluster can exhibit a multiplicity of distinct geometries, including quasicrystal-like configurations, with exotic dynamics. Linear stability analysis and dynamical simulations show that the equilibrium configurations can correspond with either stable or a type of quasistable states exhibiting periodic particle motion in the presence of frictional dissipation.
\end{abstract}

DOI: 10.1103/PhysRevB.72.085130

\section{INTRODUCTION}

While matter can modify the flow of light through scattering and absorption, light can also induce forces on matter and thus modifying its motion. It is well known that an electromagnetic wave exerts radiation pressure on matter, ${ }^{1}$ and optical gradient forces have been employed fruitfully to manipulate and trap small particles. ${ }^{2,3}$ In the last three decades, optical manipulation has been developed into a main stream laboratory tool in many different areas of science and engineering, ${ }^{4-6}$ including biology, ${ }^{7-9}$ colloidal science, ${ }^{10-15}$ and atomic physics. ${ }^{19-23}$ And yet, possible applications are far from being exhausted.

The working principle behind most of the existing applications of optical manipulation is that a strongly focused beam can trap small particles. The experimental work of Burns et al. ${ }^{13-18}$ demonstrated another weaker optical binding force between dielectric particles. Since then, this interparticle force, arising from the coherent multiple scattering of light rather than a focused beam with nonuniform intensity, has been subsequently observed in experiments of various types. ${ }^{22-29}$ Because the force alternates between attraction and repulsion as a function of particle separation, it was then suggested that such interaction can be utilized for the binding of a collection of dielectric particles. However, controversies such as whether this optical binding force is stable or not remains unsolved.

In this paper, through rigorous calculations, we explicitly identify cluster configurations of micron-sized dielectric spheres bound by coherent multiple scattering induced optical forces, and delineate their dynamics. Such a cluster of small particles behaves like "molecules," except here the scale length corresponds to the light wavelength, and the binding comes from the exchange of real photon rather than virtual photon. The force is strong enough to overcome other relevant interactions such as the van der Waals forces, gravity, and thermal fluctuations. The same number of particles can be stabilized in a large variety of distinct shapes and bond lengths, and exhibit a multiplicity of static and drifting equilibrium configurations. What is rather amazing is that the clusters can have distinct shapes and structures, and have well-defined vibration frequencies, and yet the structural order is derived from an incident electromagnetic plane wave that has uniform intensity (in contrast to gradient force that is
PACS number(s): 78.67. $-\mathrm{n}, 42.70 . \mathrm{Qs}, 45.50 .-\mathrm{j}, 78.90 .+\mathrm{t}$

derived from a nonuniform field). Since the optical force is neither a central force nor conservative, the dynamical characteristics are very different from ordinary molecules bound by chemical forces; and the equilibrium configurations can correspond with either stable or a type of quasistable states in which the cluster maintains an average shape but individual particles exhibit periodic motion in the presence of frictional dissipation. Besides theoretical interest, the concept of a photonic cluster may offer an alternative way to manipulate ultrafine particles into artificial structures.

\section{MODEL DESCRIPTION AND METHODOLOGY}

Consider a cluster of $N$ identical dielectric spheres illuminated by an incident time-harmonic electromagnetic wave

$$
\vec{E}_{i n c}(\vec{E}, t)=E_{0} \hat{x}\left[e^{i k z-i \omega t}+e^{-i k z-i \omega t}\right],
$$

consisting of two counter-propagating $x$-polarized monochromatic beams of the same intensity, with angular frequency $\omega$ and $k=\omega / c$. The two beams interfere to form fringes with intensity varying as $\sim \cos (k z)$. While the Rayleigh particle (particle with size much smaller than a wavelength) is always attracted toward the intensity maximum plane, for a particle with a size comparable to the incident wavelength, either the intensity maxima or minima are stable. If the maxima are stable, then the minima are unstable, and vice versa. In order to be definitive, in what follows the dielectric spheres have radii $r_{s}=0.414 \mu \mathrm{m}$, mass density $1050 \mathrm{~kg} / \mathrm{m}^{3}$ (mass of a sphere $m=3.1 \mathrm{fN}$ ), relative dielectric constant $\varepsilon_{r}=2.53$ (polystyrene), and the incident wavelength $\lambda=0.52 \mu \mathrm{m}$ (size parameter $k r_{s}=5$ ). For our specific parameters, we have calculated that the intensity maximum plane $(z=0$ plane $)$ is stable, and the gradient force on the sphere as a function away from the stable plane has a cosine-like profile. The maximum trapping force is about 23 $\mathrm{pN}$, about five times the transverse binding force. The amount of work to move a sphere from its stable plane (maximum intensity) to a minimum intensity plane is $\sim 460 k_{b} T$ (where $T=300 \mathrm{~K}$ denotes room temperature and $k_{b}$ is the Boltzmann constant). Thus, the gradient force along the $z$ direction traps the spheres in the $x y$ plane and we consider the sphere cluster as a two dimensional entity. 
Since the intensity of the incident light is uniform on the $x y$ plane, the forces on the spheres can only arise from multiple scattering. The light induced force (hence, the strength of optical binding) is proportional to the intensity of the incident light, set to be $0.01 \mathrm{~W} / \mu \mathrm{m}^{2}$ for each of the two beams, similar to that used in the experiments in Ref. 13. We note that the physics of the problem remains qualitatively similar when the relevant parameters (sphere size, wavelength, etc.) are varied to within an order of magnitude, or when a single plane wave is employed instead of two. In the latter all the phenomena are reproduced in the frame comoving with the cluster along the $z$ direction.

We calculate the time-averaged electromagnetic force that is responsible for optical binding. While there are other approaches, ${ }^{16,27,30,31}$ we compute the time-averaged force acting on sphere $i,\left\langle\vec{F}_{i}\right\rangle_{t}$ via a surface integral of the timeaveraged Maxwell stress tensor ${ }^{32}\langle\overleftrightarrow{T}\rangle_{t}$ over the sphere's surface

$$
\left\langle\vec{F}_{i}\right\rangle_{t}=\oiint_{\text {surface of sphere } i}\langle\overleftrightarrow{T}\rangle_{t} \cdot d \vec{S}
$$

where

$$
\langle\overleftrightarrow{T}\rangle_{t}=\frac{1}{2} \vec{E} \vec{D}^{*}+\frac{1}{2} \vec{H} \vec{B}^{*}-\frac{1}{4}\left(\vec{E} \cdot \vec{D}^{*}\right) \overleftrightarrow{I}-\frac{1}{4}\left(\vec{H} \cdot \vec{B}^{*}\right) \overleftrightarrow{I}
$$

The electromagnetic fields required in evaluating the Maxwell stress tensor are computed by an accurate multiple scattering theory, ${ }^{33}$ which is an extension of the single sphere Mie theory to a cluster of arbitrarily configured dielectric spheres. We present a brief outline of the multiple scattering theory in this section and the theory is summarized in Appendix A. The readers are also referred to Ref. 33 for a comprehensive account.

In the multiple scattering theory, the field quantities are expanded in vector spherical harmonics. The expansion coefficients are determined by numerically solving a system of $2 N L_{\max }\left(L_{\max }+2\right)$ linear equations via a variant of the biconjugate gradient method. ${ }^{34}$ Here, $L_{\max }$ is the truncation order of the series. In most cases, taking $L_{\max }$ to be the smallest integer greater than $k r_{s}+4.05\left(k r_{s}\right)^{1 / 3}+2$ is sufficient. ${ }^{33} \mathrm{In}$ some exceptional cases, a larger $L_{\max }$ is required. The convergence of the series is examined by looking at the sensitivity of the final result to increasing $L_{\max }$. For the electromagnetic force calculation on a cluster of spheres, this multiple scattering and Maxwell stress tensor method is quite possibly the most accurate and efficient method that can be applied. ${ }^{35}$

\section{RESULTS AND DISCUSSIONS}

\section{A. Forces on a pair of spheres}

Figure 1 shows the force for a two-sphere cluster with the bisphere axis tilted at $45^{\circ}$ to the polarization. The nonretarded attractive van der Waals force ${ }^{36}$ of the form

$$
F_{\text {nonretarded van der Waals }}=\frac{-A r_{s}}{12\left(R-2 r_{s}\right)^{2}}
$$

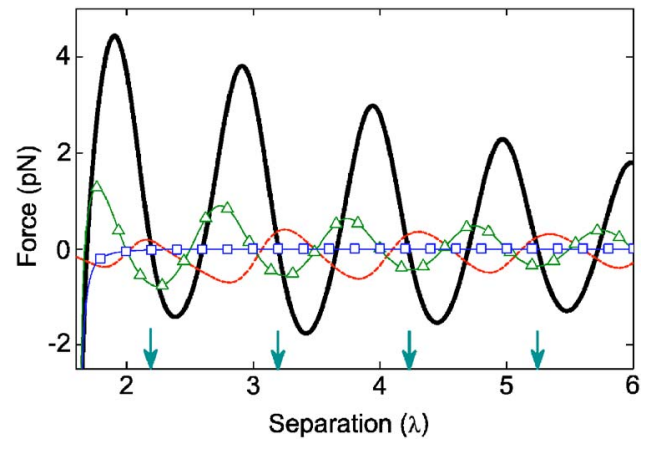

FIG. 1. (Color online) Forces acting on two-dielectric spheres, plotted as a function of their separation. The bisphere axis is tilted at $45^{\circ}$ to the polarization. The total radial force (optical plus van der Waals) and transverse force are shown by the thick solid (black) and thin dashed (red) lines, respectively. Positive radial force means repulsion while a positive transverse force means the tendency to align the bisphere chain along the incident polarization. The line with triangles (green) denotes the radial force computed from the dipole approximation (see Ref. 30) with the radiative reaction correction (see Ref. 37). Significant difference in magnitude is seen. The line with squares (blue) shows the nonretarded van der Waals force. The arrows indicate the positions of stable radial separations.

is included in the calculation, where $R$ is the separation between the centers of the spheres and $A=6.6 \times 10^{-20} \mathrm{~J}$ is the Hamaker constant. The nonretarded van der Waals force may be considered as an upper bound for the fully retarded van der Waals force. It is accurate for small separation, while retardation effects tend to weaken the van der Waals forces for large separation. From Fig. 1, it is seen that the van der Waals force is dominating only for spheres nearly in contact, while for large separation, the optical force is overwhelmingly dominant.

The alternating attractive-repulsive nature is a manifestation of the phase of the electromagnetic fields, and the radial force is seen to be stronger than the transverse force. The magnitude of the force is inversely proportional to the spheres separation in the far zone, as the scattered radiation field decays inversely from the scatterer. This long range force, on the order of a pico-Newton, dominates over the short-range attractive van der Waals force at distances over a wavelength and oscillates between attraction and repulsion with a period equal to the wavelength of the incident radiation. Stable radial positions are indicated by arrows in Fig. 1. They correspond to zero-force separations in which an increase in the radial separation would induce an attractive restoring force, whereas a reduction would induce a repulsive restoring force.

\section{B. Strength of the optical binding force in the far field}

The strength of the optical force depends on the radius of the spheres. It can be fitted asymptotically to

$$
\vec{f}\left(r_{s}\right) \cos \left[k R+\phi\left(r_{s}\right)\right] / R,
$$

where $R$ assumed to be $\gg r_{s}$. For the polarization perpendicular to the bisphere axis, $\left|\vec{f}\left(r_{s}\right)\right|$ is shown in Fig. 2. When 


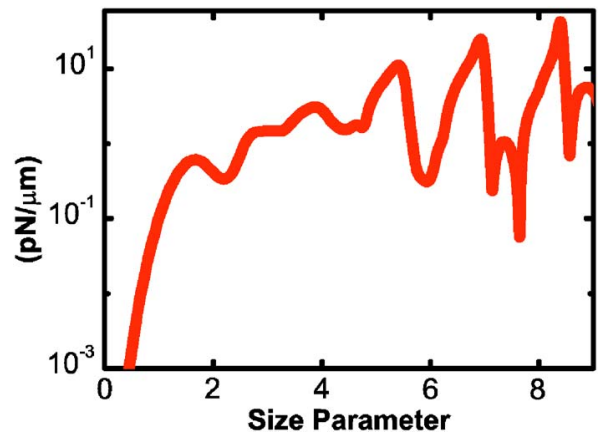

FIG. 2. (Color online) Strength of the far field optical force $\left|\vec{f}\left(r_{s}\right)\right|$. When the spheres are aligned perpendicular to the polarization of the incident light and located at each other's far field zone, the optical force asymptotically approaches $\vec{f}\left(r_{s}\right) \cos \left[k R+\phi\left(r_{s}\right)\right] / R$.

$k r_{s} \ll 1$, the force is proportional to the sixth power of the radius. For $k r_{s} \geqslant 1$ the force exhibits complex variations, as a result of resonant excitations. Although the resonance can enhance the optical force, it is not a prerequisite for optical binding and the idea of optical binding can be applied to particles of arbitrary shape. We note that the resonant optical forces for the strongly coupled optical modes were considered by some authors, ${ }^{38,39}$ whereas the resonance in Fig. 2 is for the weakly coupled spheres.

In Fig. 1, it is seen that the stable radial positions correspond to a positive aligning force along the transverse direction, therefore it is transversely unstable. The zero-force configurations can only be identified through a full two dimensional scan of the relevant forces, shown in Fig. 3. Multiple zero-force configurations exist on both the $x$ and the $y$ axes. For small separations, the force is much stronger when the bisphere axis is aligned with the polarization, due to the evanescent wave contribution. For larger separations, the optical binding force decays like $1 / R$ in general. How-

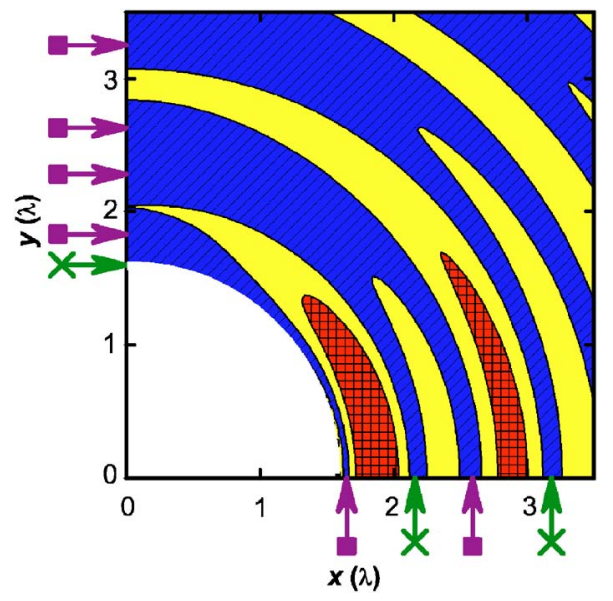

FIG. 3. (Color online) Magnitude of the optical force acting on sphere 2 located at $(x, y)$, with sphere 1 at the origin. The white region is forbidden (the spheres overlap). Blue (strip), yellow (no symbol), and red (square) corresponding to weak, medium, and strong force, respectively. Zero-force positions are marked by the arrows, with green (cross) indicating stable positions and purple (solid square) indicating unstable position. ever, it happens for the particular form of the incident beams in this work that, the force decays faster when the bisphere axis is parallel to the polarization due to the lack of propagating wave.

\section{Photonic clusters and their stability}

Zero-force configurations are not necessarily stable. Perturbation calculations are required to locate stable equilibrium configurations. For general considerations, we assume a damping force proportional to the velocity, as in most practical situations. Hence, for a cluster close to a zero-force configuration denoted by the position vector

$$
\vec{x}_{*}=\left(x_{1}, x_{2}, \ldots, x_{2 i-1}, x_{2 i}, \ldots\right),
$$

where $\left(x_{2 i-1}, x_{2 i}\right)$ denotes the zero-force coordinate $(x, y)_{i}$ of the $i$ th sphere, the linearized equation of motion is

$$
m \frac{d^{2} \Delta \vec{x}}{d t^{2}} \approx \overleftrightarrow{K} \Delta \vec{x}-b \frac{d \Delta \vec{x}}{d t},
$$

where $m$ is the sphere mass, $\left(\Delta x_{2 i-1}, \Delta x_{2 i}\right)$ is the displacement vector of the $i$ th sphere from the equilibrium, $b$ is the damping constant, and

$$
(\overleftrightarrow{K})_{j k}=\frac{\partial\left(\vec{f}_{\text {light }}\right)_{j}}{\partial \Delta x_{k}}
$$

is the force constant matrix with $\vec{f}_{\text {light }}$ being the light induced force. We consider the photonic clusters as two dimensional structures and the detailed solutions of the linearized equation of motion (7) are tabulated in Appendix B. The full three dimensional force constant matrices have been explicitly calculated for some clusters, and the stability of the cluster in the $z$ direction was always found to be much better than the other two directions. The eigenvalues of $\overleftrightarrow{K}$ dictate the stability of the cluster. Since a cluster bound by an incident light is an open system, the force is not conservative. Consequently, the force constant matrix is not necessarily symmetric, and the eigenvalues $K_{i}$ can be conjugate pairs of complex numbers, corresponding to complex valued natural frequencies, as indeed found numerically with increasing probability as the number of spheres increases.

Besides the two zero eigenvalues associated with the translation-invariant symmetry on the $x y$ plane, if all the other eigenvalues are real and negative which correspond to real valued natural frequencies, the cluster is stable. The appearance of real positive eigenvalue(s) or complex eigenvalues with $\operatorname{Re}\left(K_{i}\right)>0$ denotes instability. The eigenmodes for the complex eigenvalues are associated with spiral motions. If the damping coefficient $b=0$, one of the modes spirals inwards upon perturbation, approaching the equilibrium position, while the other eigenmode spirals outward. If $b$ is finite, then $\operatorname{Re}\left(K_{i}\right)<0$ indicates the existence of a critical damping constant

$$
b_{\text {critical }}=\sqrt{m}\left|\operatorname{Im}\left(K_{i}\right)\right| / \sqrt{\left|\operatorname{Re}\left(K_{i}\right)\right|},
$$

at which the modes become stable for $b>b_{\text {critical }}$. We denote this type of complex modes as quasistable modes. The ap- 

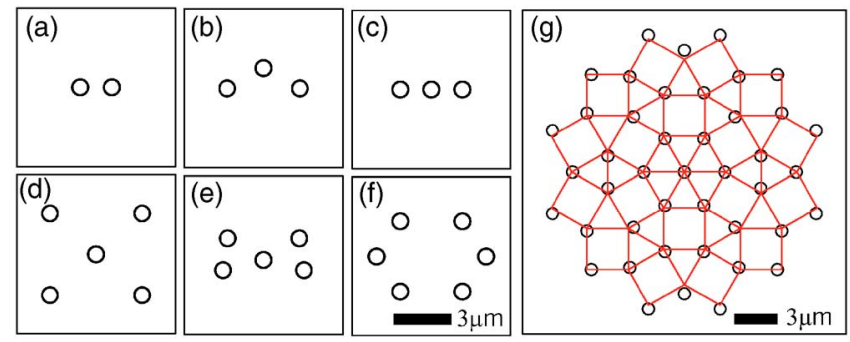

FIG. 4. (Color online) Examples of photonic clusters. The figures are drawn to scale. Configurations (b) and (e) are in drifting equilibrium (see text), and the others are in static equilibrium. All eigenmodes of configurations (a)-(e) are stable, and (f)-(g) have either stable or quasistable modes. Polarization of the incident light beam is in the horizontal direction. (g) shows a 43 -sphere photonic cluster. The lines (red) are drawn to unveil the 12-fold symmetric, square-triangle quasicrystal tiling.

pearance of one or more pairs of complex eigenvalues with $\operatorname{Re}\left(K_{i}\right)<0$, with all the rest real and negative, denotes the cluster to be quasistable, i.e., potentially stable in the presence of damping.

For a multisphere cluster, the optical force can bind the spheres into a multiplicity of distinct geometries. In Fig. 4, the stable configurations are shown in panels (a)-(e), and quasistable configurations are shown in panels (f) and (g). For the particular stable configuration shown in Fig. 4(a), the radial oscillation frequency is $540 \mathrm{kHz}$ ( $k T$ of energy, implies a fluctuation amplitude $\sim 14 \mathrm{~nm}$ ), whereas the rotational oscillation frequency is $51 \mathrm{kHz}$ ( $k T$ of energy implies a fluctuation amplitude $\sim 0.09 \mathrm{rad})$. Note that the vibration amplitude of the rotational oscillation is greater than that of the radial oscillation, if they are given the same amount of energy. This fact is also evident from the relative magnitude between the radial and transverse optical force, as shown in Fig. 1.

Comparison with molecules bound by chemical forces yields some interesting differences. First, atomic clusters can have multiple stable isomers, but the bond length always has a narrow distribution. For photonic clusters, a fixed number of spheres can be bounded into a great number of stable configurations, each of a different bound length and shape. Second, chemically bound molecules can rotate freely in space while photonic clusters, owing to the vector nature of light, are pinned in the two dimensional plane with a fixed orientation (relative to the light polarization). Third, for clusters without inversion symmetry, photon scatterings are biased in one direction and hence the cluster absorbs light momentum, leading to "drifting" equilibrium states in which the cluster moves as a stable entity in the $x y$ plane. Accordingly, the configurations shown in Figs. 4(b) and 4(e) are in drifting equilibrium while all the others are in static equilibrium. We note that for the drifting equilibriums introduced in this work, the same perturbation analysis of (6)-(8) can still be applied except now $\Delta \vec{x}$ denotes the displacement away from the drifting equilibrium that is moving with time, as shown in Appendix B.

Although the input light field is homogeneous on the $x y$ plane, it is rather intriguing that the optical binding force can

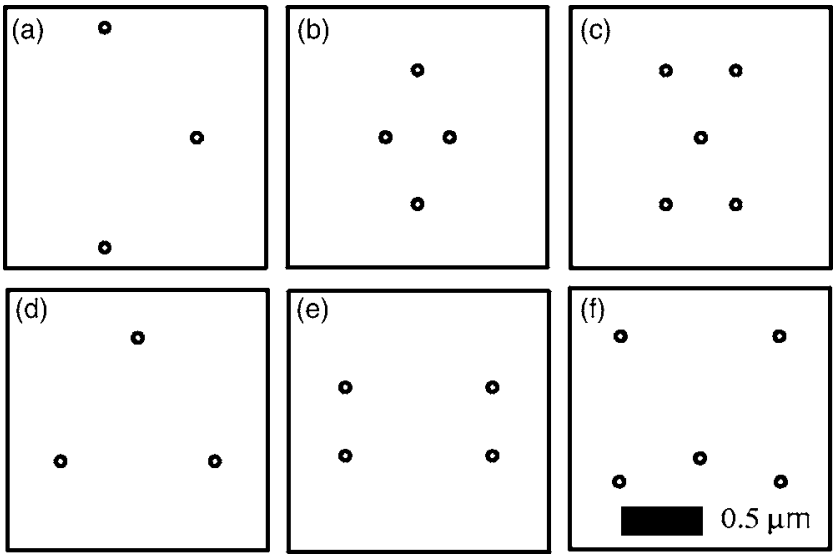

FIG. 5. Examples of photonic clusters composed of Rayleigh particles (spheres). The radius of the spheres is $33.1 \mathrm{~nm}$ (size parameter $=0.4$ ) and their dielectric constant is 2.53 . The figures are drawn to scale. Configurations (a), (d), and (f) are in drifting equilibrium (see text), and the others are in static equilibrium. All configurations are stable. Polarization of the incident light beam is in the horizontal direction.

arrange dielectric spheres into rather complex geometriesFig. 4(g) shows a 43-sphere cluster with a quasicrystal-like geometry (red lines show the exact square-triangle quasicrystal tiling). The particular configuration shown has 18 pairs of complex eigenvalues with their corresponding $b_{\text {critical }}$ between 1.3 and $17 \mathrm{pN} / \mathrm{ms}^{-1}$. Thus if $b>17 \mathrm{pN} / \mathrm{ms}^{-1}$, a stable quasicrystal-like cluster can be realized.

The optical binding force can also be utilized for binding Rayleigh particles whose size is small compared with the wavelength, some examples of such clusters are shown in Fig. 5. Note that while the force is nonconservative for large spheres, in the limit of vanishing size, the interparticle force is mainly the conservative gradient force. Consequently, the vibration frequencies for the photonic clusters composed of Rayleigh particles are always real and the quasistable modes are not found.

\section{Thermal stability}

To investigate the thermal stability of the (stable) photonic clusters, we move the spheres apart along the path of their lowest frequency eigenmode, until the projection of the restoring force onto the eigenmode switches sign. For an open system, the work done is path dependent. Here we give a plausible estimate to the depth of the equilibrium well. For typical photonic clusters the required dissociation energy per sphere was evaluated to be hundreds of $k_{b} T$, for example, the cluster in Fig. 4(a) (two spheres at distance $=3.16 \lambda$ ) has a dissociation energy of $110 k_{b} T$. Thus the photonic clusters are expected to be stable against thermal fluctuations.

\section{E. Dynamics of light bounded structures}

To study the dynamics of the quasistable photonic clusters, it is necessary to go beyond the linear stability analysis, especially for $b \approx b_{\text {critical }}$ where the point is nonhyperbolic (the linear term vanishes) and thus the higher order terms are 


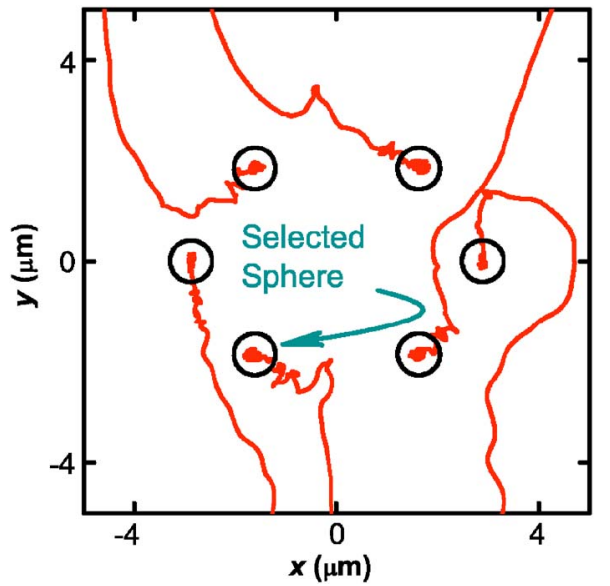

FIG. 6. (Color online) Complex mode (center-of-mass) trajectories for the cluster shown in Fig. 4(f) at $b=0$ (no damping), the cluster breaks up.

important. ${ }^{40}$ We integrate the equations of motion using an adaptive time-step Runge-Kutta-Verner algorithm. The procedures are similar to molecular dynamics simulations, except that the interparticle forces are now the light-induced forces. To illustrate the rich phenomena in dynamics, we study a six-sphere photonic cluster shown in Fig. 4(f), which has all real negative eigenvalues except for one complex conjugate pair. We ignored van der Waals forces in the following calculations since the spheres are about $3 \mu \mathrm{m}$ apart, and at that distance, the optical binding force is about 3000 times stronger than the van der Waals force. Figures 6-9
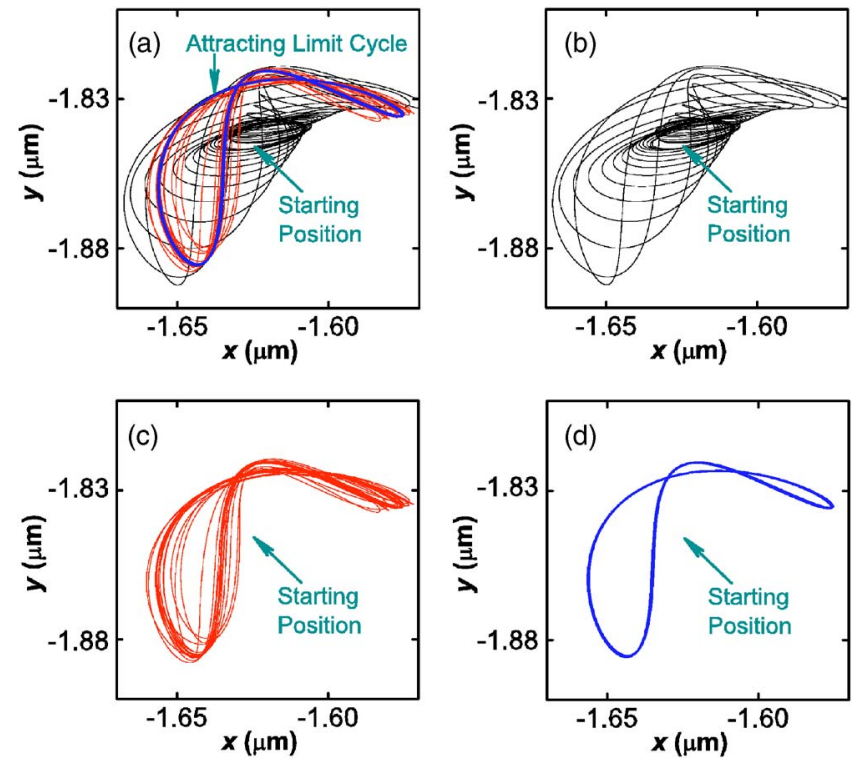

FIG. 7. (Color online) Complex mode (center-of-mass) trajectories for the selected sphere marked in Fig. 6 at $b=3.71 \mathrm{pN} / \mathrm{ms}^{-1}$. (a) The trajectory in three consecutive $0.5 \mathrm{~ms}$ intervals. The starting position of the sphere is marked on the graphs. (b) The trajectory in the first $0.5 \mathrm{~ms}$ interval. (c) The trajectory in the second $0.5 \mathrm{~ms}$ interval. (d) The trajectory in the third $0.5 \mathrm{~ms}$ interval. The cluster is attracted towards a periodic orbit with period $=32 \mu \mathrm{s}$. The size of the periodic orbit is noted to be small compared to the interparticle separations.
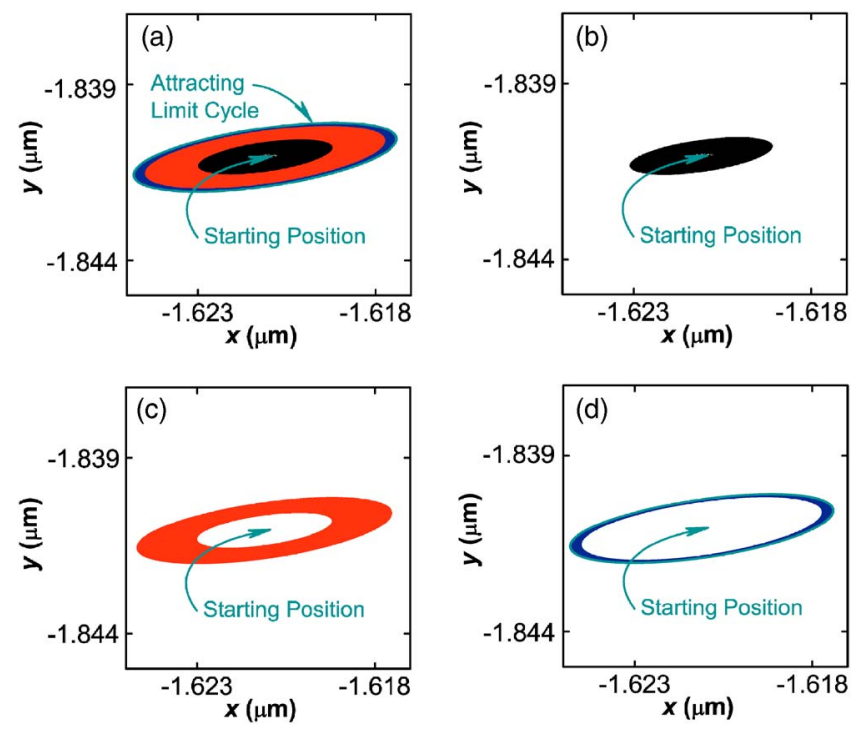

FIG. 8. (Color online) Complex mode (center-of-mass) trajectories for the selected sphere marked in Fig. 6 at $b$ $=4.31 \mathrm{pN} / \mathrm{ms}^{-1}\left(b_{\text {critical }}=4.32 \mathrm{pN} / \mathrm{ms}^{-1}\right)$. (a) The trajectory in three consecutive $130 \mathrm{~ms}$ intervals. The starting position of the sphere is marked on the graphs. (b) The trajectory in the first $130 \mathrm{~ms}$ interval. (c) The trajectory in the second $130 \mathrm{~ms}$ interval. (d) The trajectory in the third $130 \mathrm{~ms}$ interval. The cluster is attracted towards an elliptic periodic orbit with period $=15 \mu \mathrm{s}$. The size of the periodic orbit is noted to be small compared to the interparticle separations.

show the cluster dynamics at different levels of damping. For $b>b_{\text {critical }}$, each sphere exhibits damped oscillations that settle into the stable zero-force position (Fig. 9). For $b$ slightly less than $b_{\text {critical }}$, the zero-force position turns un-
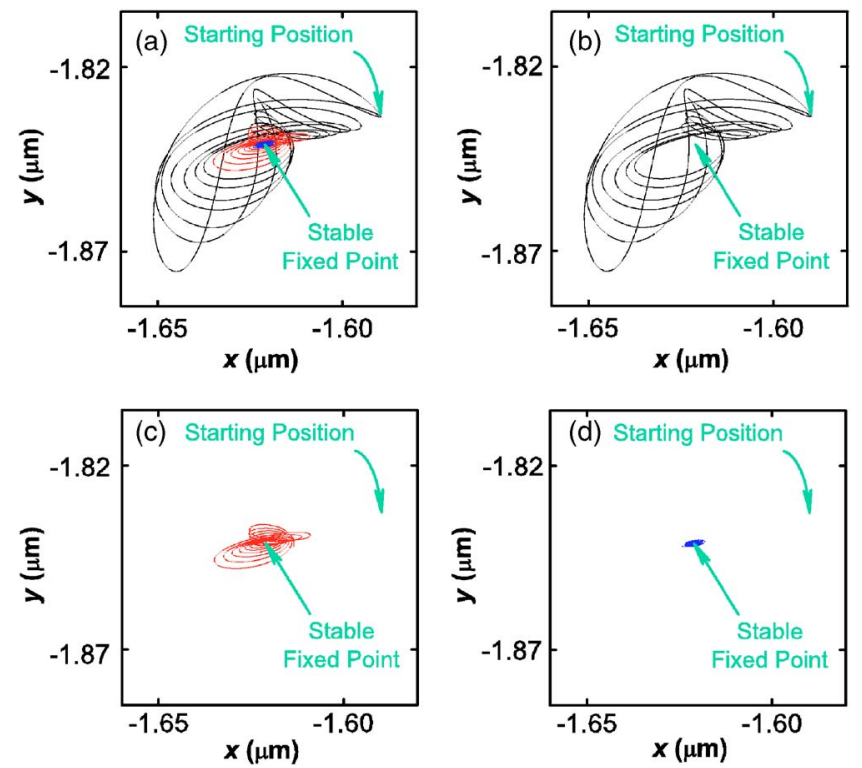

FIG. 9. (Color online) Complex mode (center-of-mass) trajectories for the selected sphere marked in Fig. 6 at $b=9.28 \mathrm{pN} / \mathrm{ms}^{-1}$. (a) The trajectory in three consecutive $0.2 \mathrm{~ms}$ intervals. The starting position of the sphere is marked on the graphs. (b) The trajectory in the first $0.2 \mathrm{~ms}$ interval. (c) The trajectory in the second $0.2 \mathrm{~ms}$ interval. (d) The trajectory in the third $0.2 \mathrm{~ms}$ interval. The cluster is damped towards the stable zero-force position. 
stable and an attracting elliptic periodic orbit is formed (Fig. 8 , period $=15 \mu \mathrm{s}$ ) for each sphere in the immediate neighborhood of the zero-force position. This periodic dynamics arise because the repulsive part of the force becomes less than that predicted by the linear stability analysis as the sphere spirals outward, allowing an orbit to be established. Here the damping dissipation is counterbalanced by the incident light energy. Hence, from the dynamics perspective $b_{\text {critical }}$ is a supercritical Hopf bifurcation point. ${ }^{40} \mathrm{~A}$ further decrease in $b$ enlarges and deforms the periodic orbit (Fig. 7, period $=32 \mu \mathrm{s})$, and additional decrease in damping leads to instability (Fig. 6).

\section{SUMMARY AND CONCLUSION}

In summary, we showed using a rigorous multiplescattering technique that the homogeneous light-field of an incident plane wave can organize small particles into stable clusters. These clusters have well-defined geometry (bond length and angle) and well-defined vibration frequencies, and they are bound by light, and hence, we call them photonic clusters. Both the static and dynamic properties are rather interesting, which is a manifestation that we have an open system, in contrast to chemical molecules that are bound by conservative forces. Through molecular dynamics simulation, the quasistable photonic clusters associated with complex vibration frequencies are investigated. The stability of the quasistable photonic clusters depends upon the damping provided by the environment; as the damping increases from zero to beyond $b_{\text {critical }}$, the initially unstable equilibrium transforms into a limit cycle, and then a stable equilibrium.

\section{ACKNOWLEDGMENTS}

Support by RGC Hong Kong through HKUST6138/00P is gratefully acknowledged. Z.F.L. is supported by CNKBRSF, NSFC through 10474014 and 10321003 and PCSIRT. We thank L. M. Li for help in the molecular dynamics code.

\section{APPENDIX A: MULTIPLE SCATTERING THEORY FOR CLUSTERS OF SPHERES}

We present a summary of the standard multiple scattering theory. The theory has been discussed in the literature and our approach and notations are closely parallel to that of Ref. 33.

\section{Complete and orthogonal solutions of the wave equation: The vector spherical harmonics}

The electromagnetic scattering problem involves solving the time-harmonic vector wave equation

$$
\nabla \times \nabla \times \vec{E}-k^{2} \vec{E}=\overrightarrow{0},
$$

where $k$ is the wave number. The time dependence is assumed to be $e^{-i \omega t}$ and will be omitted in the remaining text. Once the electric field is obtained from (A1), the magnetic field can then be evaluated as

$$
\vec{B}=\nabla \times \vec{E} / i \omega
$$

In a homogeneous region, the complete and orthogonal set of solutions to the wave equation (A1), in spherical coordinates, are the vector spherical harmonics

$$
\begin{aligned}
\vec{M}_{m n}^{(J)}(k r)= & {\left[i \pi_{m n}(\cos \theta) \hat{\theta}-\tau_{m n}(\cos \theta) \hat{\phi}\right] z_{n}^{J}(k r) e^{i m \phi}, } \\
\vec{N}_{m n}^{(J)}(k r)= & {\left[\tau_{m n}(\cos \theta) \hat{\theta}+i \pi_{m n}(\cos \theta) \hat{\phi}\right](1 / k r) d / d(k r) } \\
& \times\left[(k r) z_{n}^{J}(k r)\right] e^{i m \phi}+n(n+1) P_{n}^{m}(\cos \theta) \\
& \times\left[z_{n}^{J}(k r) / k r\right] e^{i m \phi} \hat{r},
\end{aligned}
$$

where $\{\hat{r}, \hat{\theta}, \hat{\phi}\}$ are the unit vectors in the spherical coordinate system, and the vector spherical harmonics are related to each other by

$$
\begin{aligned}
& \vec{M}_{m n}^{(J)}(k r)=(1 / k) \nabla \times \vec{N}_{m n}^{(J)}(k r), \\
& \vec{N}_{m n}^{(J)}(k r)=(1 / k) \nabla \times \vec{M}_{m n}^{(J)}(k r) .
\end{aligned}
$$

In (A3), $z_{n}^{J}$ specifies the radial dependence and is appropriately selected from any of the four functions

$$
z_{n}^{J}=\left\{\begin{array}{l}
j_{n} \text { if } J=1, \\
y_{n} \text { if } J=2, \\
h_{n}^{(1)} \text { if } J=3, \\
h_{n}^{(2)} \text { if } J=4,
\end{array}\right.
$$

where $j_{n}$ is the spherical Bessel function, $y_{n}$ is the spherical Neumann function, and $h_{n}^{(1)}$ and $h_{n}^{(2)}$ are the Hankel spherical function of the first and second kind, respectively. The angular dependent functions are defined as

$$
\begin{gathered}
\pi_{m n}(\cos \theta)=\frac{m}{\sin \theta} P_{n}^{m}(\cos \theta), \\
\tau_{m n}(\cos \theta)=\frac{d}{d \theta} P_{n}^{m}(\cos \theta),
\end{gathered}
$$

where

$$
P_{n}^{m}(x)=\frac{1}{2^{n} n !}\left(1-x^{2}\right)^{m / 2} \frac{d^{n+m}}{d x^{n+m}}\left[\left(x^{2}-1\right)^{n}\right]
$$

is the associated Legendre function of the first kind and of degree $n(=1,2,3 \ldots)$ and order $m(=0, \pm 1, \pm 2, \ldots \pm n)$. In the Mie scattering theory for a single sphere, the vector spherical harmonics (A3) are the quasinormal modes for the electromagnetic field.

\section{Coupled linear equations for the interactive scattering coefficients}

The multiple scattering theory deals with the electromagnetic scattering problem of a collection of isotropic, homogeneous spheres under the illumination of a monochromatic incident wave. Consider a cluster of $N$ spheres, where sphere $j$ has dielectric constant $\varepsilon_{j}$ and permeability $\mu_{j}$ and those of the background medium are $\varepsilon$ and $\mu$, respectively. Within a homogeneous region, the complete and orthogonal set of so- 
lutions to wave equation in spherical coordinates are the vector spherical harmonics (A3), thus an arbitrary initial incident wave can be decomposed into a series of the vector spherical harmonics

$$
\vec{E}_{0}\left(\vec{r}_{j}\right)=-\sum_{n=1}^{\infty} \sum_{m=-n}^{n} i E_{m n}\left[p_{m n}^{(j \rightarrow j)} \vec{N}_{m n}^{(1)}\left(k \vec{r}_{j}\right)+q_{m n}^{(j \rightarrow j)} \vec{M}_{m n}^{(1)}\left(k \vec{r}_{j}\right)\right],
$$

where $\vec{r}_{j}$ is the vector pointing from the sphere's center to the observation point,

$$
E_{m n}=E_{0} i^{n}(2 n+1) \frac{(n-m) !}{(n+m) !},
$$

and $\left\{p_{m n}^{(j \rightarrow j)}, q_{m n}^{(j \rightarrow j)}\right\}$ are the expansion coefficients for the initial incident wave, and are assumed to be known for the problem under consideration.

By expanding the total incident field that strikes the surface of the $j$ th sphere $\vec{E}_{\text {inc }}^{(j)}$, the scattered field $\vec{E}_{\text {sca }}^{(j)}$, and the internal field $\vec{E}_{\text {int }}^{(j)}$, as series of the vector spherical harmonics in the coordinate frame center at sphere $j$, and then apply the standard boundary conditions

$$
\begin{aligned}
& \left\{\vec{E}_{\text {inc }}\left(\vec{r}_{j}\right)+\vec{E}_{\text {sca }}\left(\vec{r}_{j}\right)-\vec{E}_{\text {int }}\left(\vec{r}_{j}\right)\right\} \times \hat{r}_{j}=\overrightarrow{0}, \\
& \left\{\vec{H}_{\text {inc }}\left(\vec{r}_{j}\right)+\vec{H}_{\text {sca }}\left(\vec{r}_{j}\right)-\vec{H}_{\text {int }}\left(\vec{r}_{j}\right)\right\} \times \hat{r}_{j}=\overrightarrow{0},
\end{aligned}
$$

over the surface of sphere, after some algebras, one arrives at

$$
\begin{gathered}
\vec{E}_{\mathrm{inc}}^{(j)}=-\sum_{n=1}^{\infty} \sum_{m=-n}^{n} i E_{m n}\left[p_{m n}^{(j)} \vec{N}_{m n}^{(1)}+q_{m n}^{(j)} \vec{M}_{m n}^{(1)}\right], \\
\vec{E}_{\mathrm{sca}}^{(j)}=\sum_{n=1}^{\infty} \sum_{m=-n}^{n} i E_{m n}\left[a_{n}^{(j)} p_{m n}^{(j)} \vec{N}_{m n}^{(3)}+b_{n}^{(j)} q_{m n}^{(j)} \vec{M}_{m n}^{(3)}\right], \\
\vec{E}_{\mathrm{int}}^{(j)}=\sum_{n=1}^{\infty} \sum_{m=-n}^{n} i E_{m n}\left[d_{n}^{(j)} p_{m n}^{(j)} \vec{N}_{m n}^{(1)}+c_{n}^{(j)} q_{m n}^{(j)} \vec{M}_{m n}^{(1)}\right],
\end{gathered}
$$

where

$$
\begin{gathered}
a_{n}^{(j)}=\frac{\mu m_{j}^{2} j_{n}\left(m_{j} x_{j}\right)\left[x_{j} j_{n}\left(x_{j}\right)\right]^{\prime}-\mu_{j} j_{n}\left(x_{j}\right)\left[m_{j} x_{j} j_{n}\left(m_{j} x_{j}\right)\right]^{\prime}}{\mu m_{j}^{2} j_{n}\left(m_{j} x_{j}\right)\left[x_{j} h_{n}^{(1)}\left(x_{j}\right)\right]^{\prime}-\mu_{j} h_{n}^{(1)}\left(x_{j}\right)\left[m_{j} x_{j} j_{n}\left(m_{j} x_{j}\right)\right]^{\prime}}, \\
b_{n}^{(j)}=\frac{\mu_{j} j_{n}\left(m_{j} x_{j}\right)\left[x_{j} j_{n}\left(x_{j}\right)\right]^{\prime}-\mu j_{n}\left(x_{j}\right)\left[m_{j} x_{j} j_{n}\left(m_{j} x_{j}\right)\right]^{\prime}}{\mu_{j} j_{n}\left(m_{j} x_{j}\right)\left[x_{j} h_{n}^{(1)}\left(x_{j}\right)\right]^{\prime}-\mu h_{n}^{(1)}\left(x_{j}\right)\left[m_{j} x_{j} j_{n}\left(m_{j} x_{j}\right)\right]^{\prime}}, \\
c_{n}^{(j)}=\frac{\mu_{j} j_{n}\left(x_{j}\right)\left[x_{j} h_{n}^{(1)}\left(x_{j}\right)\right]^{\prime}-\mu_{j} h_{n}^{(1)}\left(x_{j}\right)\left[x_{j} j_{n}\left(x_{j}\right)\right]^{\prime}}{\mu_{j} j_{n}\left(m_{j} x_{j}\right)\left[x_{j} h_{n}^{(1)}\left(x_{j}\right)\right]^{\prime}-\mu h_{n}^{(1)}\left(x_{j}\right)\left[m_{j} x_{j} j_{n}\left(m_{j} x_{j}\right)\right]^{\prime}}, \\
d_{n}^{(j)}=\frac{\mu_{j} m_{j} j_{n}\left(x_{j}\right)\left[x_{j} h_{n}^{(1)}\left(x_{j}\right)\right]^{\prime}-\mu_{j} m_{j} h_{n}^{(1)}\left(x_{j}\right)\left[x_{j} j_{n}\left(x_{j}\right)\right]^{\prime}}{\mu m_{j}^{2} j_{n}\left(m_{j} x_{j}\right)\left[x_{j} h_{n}^{(1)}\left(x_{j}\right)\right]^{\prime}-\mu_{j} h_{n}^{(1)}\left(x_{j}\right)\left[m_{j} x_{j} j_{n}\left(m_{j} x_{j}\right)\right]^{\prime}},
\end{gathered}
$$

are the ordinary Mie coefficients for a single sphere, the prime denotes a differentiation with respect to the argument in the parentheses, $x_{j}=k_{j} a_{j}$ is the size parameter of sphere $j$ with $a_{j}$ the radius of sphere $j$, and $m_{j}=k_{j} / k$. Note that in our terminology, the initial incident wave (A8) is different from the total incident wave (A11). The only unknown quantities in (A11)-(A13) are $\left\{p_{m n}^{(j)}, q_{m n}^{(j)}\right\}$, which are the expansion coefficients for the total incident field striking each sphere.

An alternative expression for the total incident field of each sphere can be obtained by noting that these quantities consist of two distinct parts, firstly the initial incident wave striking the whole cluster $\vec{E}_{0}$, and secondly the sum of all the scattered wave from other spheres $\Sigma_{l \neq j} \vec{E}_{\text {sca. }}^{(l)}$ Invoking the translation addition theorem for the vector spherical harmonics (A19), one arrives at

$$
\begin{aligned}
\vec{E}_{\mathrm{inc}}^{(j)}= & \vec{E}_{0}+\sum_{l \neq j}^{(1, N)} \vec{E}_{\mathrm{sca}}^{(l)}=-\sum_{l=1}^{N} \sum_{n=1}^{\infty} \sum_{m=-n}^{n} i E_{m n}\left[p_{m n}^{(l \rightarrow j)} \vec{N}_{m n}^{(1)}\left(k \vec{r}_{j}\right)\right. \\
& \left.+q_{m n}^{(l \rightarrow j)} \vec{M}_{m n}^{(1)}\left(k \vec{r}_{j}\right)\right],
\end{aligned}
$$

where

$$
\begin{aligned}
& p_{m n}^{(l \rightarrow j)}=-\sum_{v=1}^{\infty} \sum_{u=-v}^{v}\left[\begin{array}{l}
A_{m n}^{u v(3)}(l \rightarrow j) a_{v}^{(l)} p_{u v}^{(l)} \\
+B_{m n}^{u v(3)}(l \rightarrow j) b_{v}^{(l)} q_{u v}^{(l)}
\end{array}\right], \\
& q_{m n}^{(l \rightarrow j)}=-\sum_{v=1}^{\infty} \sum_{u=-v}^{v}\left[\begin{array}{l}
B_{m n}^{u v(3)}(l \rightarrow j) a_{v}^{(l)} p_{u v}^{(l)} \\
+A_{m n}^{u v(3)}(l \rightarrow j) b_{v}^{(l)} q_{u v}^{(l)}
\end{array}\right],
\end{aligned}
$$

for $(l \neq j)$, represent the scattered wave of sphere $l$ expanded in the coordinate system centered at sphere $j$, with the coordinate axes of the two systems being parallel to each other, and $\left\{A_{m n}^{u v(3)}(l \rightarrow j), B_{m n}^{u v(3)}(l \rightarrow j)\right\}$ are the normalized translation coefficients defined in (A25).

Now, equating (A11) and (A15), and with the help of (A16) and the orthogonality of the vector spherical harmonics, we arrive at a set of linear equations for the interactive scattering coefficients

$$
\begin{aligned}
p_{m n}^{(j)}= & \sum_{l=1}^{N} p_{m n}^{(l \rightarrow j)}=p_{m n}^{(j \rightarrow j)} \\
& -\sum_{l \neq j}^{(1, N)} \sum_{v=1}^{\infty} \sum_{u=-v}^{v}\left[\begin{array}{l}
A_{m n}^{u v(3)}(l \rightarrow j) a_{v}^{(l)} p_{u v}^{(l)} \\
+B_{m n}^{u v(3)}(l \rightarrow j) b_{v}^{(l)} q_{u v}^{(l)}
\end{array}\right], \\
q_{m n}^{(j)}= & \sum_{l=1}^{N} q_{m n}^{(l \rightarrow j)}=q_{m n}^{(j \rightarrow j)} \\
& -\sum_{l \neq j}^{(1, N)} \sum_{v=1}^{\infty} \sum_{u=-v}^{v}\left[\begin{array}{l}
B_{m n}^{u v(3)}(l \rightarrow j) a_{v}^{(l)} p_{u v}^{(l)} \\
+A_{m n}^{u v(3)}(l \rightarrow j) b_{v}^{(l)} q_{u v}^{(l)}
\end{array}\right] .
\end{aligned}
$$

Equation (A17) is the main equation for the multiple scattering theory.

The infinite series sum of the angular momentum index $v$ in (A17) can be truncated at a finite value $L_{\max }{ }^{33}$ as evident from the fact that the single sphere Mie coefficients $a_{n}^{(l)}$ approach zero for $n \gg x_{l}$. Thus 


$$
\begin{gathered}
p_{m n}^{(j)}=p_{m n}^{(j \rightarrow j)}-\sum_{l \neq j}^{(1, N)} \sum_{v=1}^{L_{\max }} \sum_{u=-v}^{v}\left[\begin{array}{l}
A_{m n}^{u v(3)}(l \rightarrow j) a_{v}^{(l)} p_{u v}^{(l)} \\
+B_{m n}^{u v(3)}(l \rightarrow j) b_{v}^{(l)} q_{u v}^{(l)}
\end{array}\right], \\
q_{m n}^{(j)}=q_{m n}^{(j \rightarrow j)}-\sum_{l \neq j}^{(1, N)} \sum_{v=1}^{L_{\max }} \sum_{u=-v}^{v}\left[\begin{array}{l}
B_{m n}^{u v(3)}(l \rightarrow j) a_{v}^{(l)} p_{u v}^{(l)} \\
+A_{m n}^{u v(3)}(l \rightarrow j) b_{v}^{(l)} q_{u v}^{(l)}
\end{array}\right] .
\end{gathered}
$$

Equation (A18) is a linear system of $2 N L_{\max }\left(L_{\max }+2\right)$ equations, which can be solved numerically by standard technique such as direct matrix inversion or iterative algorithms like the conjugate gradient method. In our calculations, convergence can usually be achieved by taking $L_{\max }$ to be the smallest integer greater than $k r_{s}+4.05\left(k r_{s}\right)^{1 / 3}+2 .^{33}$ In some exceptional cases where the interactions of the spheres are strong, a larger $L_{\max }$ is required, especially when resonances are triggered. 39

\section{Translation coefficients for vector spherical harmonics}

A crucial step in the multiple scattering theory is to match the boundary conditions over the surface of every sphere. The task was accomplished by the translation addition theorem of the vector spherical harmonics

$$
\begin{aligned}
& \vec{M}_{u v}^{(j)}\left(k \vec{r}_{l}\right)=\sum_{n=0}^{\infty} \sum_{m=-n}^{n}\left[\begin{array}{c}
\widetilde{A}_{m n}^{u v(J)}(l \rightarrow j) \vec{M}_{m n}^{(1)}\left(k \vec{r}_{j}\right) \\
+\widetilde{B}_{m n}^{u v(J)}(l \rightarrow j) \vec{N}_{m n}^{(1)}\left(k \vec{r}_{j}\right)
\end{array}\right], \\
& \vec{N}_{u v}^{(J)}\left(k \vec{r}_{l}\right)=\sum_{n=0}^{\infty} \sum_{m=-n}^{n}\left[\begin{array}{c}
\widetilde{B}_{m n}^{u v(J)}(l \rightarrow j) \vec{M}_{m n}^{(1)}\left(k \vec{r}_{j}\right) \\
+\widetilde{A}_{m n}^{u v(J)}(l \rightarrow j) \vec{N}_{m n}^{(1)}\left(k \vec{r}_{j}\right)
\end{array}\right],
\end{aligned}
$$

for $r<d_{l j}$, and

$$
\begin{aligned}
& \vec{M}_{u v}^{(J)}\left(k \vec{r}_{l}\right)=\sum_{n=0}^{\infty} \sum_{m=-n}^{n}\left[\begin{array}{c}
\widetilde{A}_{m n}^{u v(1)}(l \rightarrow j) \vec{M}_{m n}^{(J)}\left(k \vec{r}_{j}\right) \\
+\widetilde{B}_{m n}^{u v(1)}(l \rightarrow j) \vec{N}_{m n}^{(J)}\left(k \vec{r}_{j}\right)
\end{array}\right], \\
& \vec{N}_{u v}^{(J)}\left(k \vec{r}_{l}\right)=\sum_{n=0}^{\infty} \sum_{m=-n}^{n}\left[\begin{array}{c}
\widetilde{B}_{m n}^{u v(1)}(l \rightarrow j) \vec{M}_{m n}^{(J)}\left(k \vec{r}_{j}\right) \\
+\widetilde{A}_{m n}^{u v(1)}(l \rightarrow j) \vec{N}_{m n}^{(J)}\left(k \vec{r}_{j}\right)
\end{array}\right],
\end{aligned}
$$

for $r>d_{l j}$, where $\widetilde{A}_{m n}^{u v(J)}(l \rightarrow j)$ and $\widetilde{B}_{m n}^{u v(J)}(l \rightarrow j)$ are the translation coefficients for the transformation from the origin centered at sphere $l$ to that of sphere $j$, with the coordinate axes in both systems being parallel to each other. Their expressions are

$$
\begin{aligned}
\widetilde{A}_{m n}^{u v(J)}(l \rightarrow j) & =(-1)^{u} i^{v-n}(2 v+1) /[2 v(v+1)] \\
& \times \sum_{p=|n-v|}^{n+v}(-i)^{p}[n(n+1)+v(v+1)-p(p+1)] \\
& \times a(m, n,-u, v, p) z_{p}^{J}\left(k d_{l j}\right) P_{p}^{m-u}\left(\cos \theta_{l j}\right) e^{i(m-u) \phi_{l j}},
\end{aligned}
$$

$$
\begin{aligned}
\widetilde{B}_{m n}^{u v(J)}(l \rightarrow j) & =(-1)^{u} i^{v-n}(2 v+1) /[2 v(v+1)] \\
& \times \sum_{p=|n-v|}^{n+v}(-i)^{p} b(m, n,-u, v, p, p-1) \\
& \times z_{p}^{J}\left(k d_{l j}\right) P_{p}^{m-u}\left(\cos \theta_{l j}\right) e^{i(m-u) \phi_{l j}},
\end{aligned}
$$

where $\left\{d_{l j}, \theta_{l j}, \phi_{l j}\right\}$ are the spherical coordinates of the vector pointing from the center of sphere $l$ to that of the sphere $j$ and

$$
\begin{aligned}
a(m, n, u, v, p)= & {[(2 p+1) / 2](p-m-u) ! /(p+m+u) ! } \\
& \times \int_{-1}^{1} P_{n}^{m}(x) P_{v}^{u}(x) P_{p}^{m+u}(x) d x,
\end{aligned}
$$

$$
\begin{aligned}
& b(m, n,-u, v, p, p-1) \\
& =(2 p+1) /(2 p-1) \\
& \times\left[\begin{array}{l}
(v-u)(v+u+1) a(m, n,-u-1, v, p-1) \\
-(p-m+u)(p-m+u-1) \\
\times a(m, n,-u+1, v, p-1) \\
+2 u(p-m+u) a(m, n,-u, v, p-1)
\end{array}\right]
\end{aligned}
$$

For convenience, the normalized translation coefficients are defined to be

$$
\begin{aligned}
& A_{m n}^{u v(J)}(l \rightarrow j)=\left(E_{u v} / E_{m n}\right) \widetilde{A}_{m n}^{u v(J)}(l \rightarrow j), \\
& B_{m n}^{u v(J)}(l \rightarrow j)=\left(E_{u v} / E_{m n}\right) \widetilde{B}_{m n}^{u v(J)}(l \rightarrow j) .
\end{aligned}
$$

The derivation of the translation addition theorem is rather lengthy and the interested readers are referred to Refs. 41-43. In actual numerical implementation, instead of directly applying the formulas listed above, it is often far more efficient to evaluate the translation coefficients with some recursive algorithms. ${ }^{43}$ The trick of "symmetry and rotational decomposition" discussed in Ref. 44 is also very useful.

\section{APPENDIX B: LINEAR STABILITY ANALYSIS OF PHOTONIC CLUSTERS}

It was shown in the text that a cluster of dielectric particles can be organized into stable geometric structures by a plane wave. Two types of equilibrium for the photonic clusters were defined in the text, the static equilibrium where the force on each sphere is zero, and the drifting equilibrium where the force on each sphere is not zero but identical. For the drifting equilibrium, the shape of the cluster will remain fixed but the whole cluster will move along the direction of the optical force. The static equilibrium may be considered as a special case of the drifting equilibrium with the drifting force identically equal to zero. Photonic clusters possessing inversion symmetry must be in static equilibrium, while for clusters without inversion symmetry, photon scatterings are biased in one direction and hence the cluster absorbs light momentum, leading to "drifting" equilibrium states in which the cluster moves as a stable entity in the $x y$ plane. The 
stability of the photonic clusters was investigated by a linear stability analysis and the details are summarized in this section.

We first consider the linearized equation of motion for a photonic cluster consists of $N$ particles that are trapped on the $x y$ plane by the incident field (1). Denoting the displacement of the particles away from the equilibrium position by the vector

$$
\Delta \vec{x}=\left(\Delta x_{1}, \Delta y_{1}, \Delta x_{2}, \Delta y_{2}, \ldots, \Delta x_{i}, \Delta y_{i}, \ldots\right),
$$

then the equation of motion governing the motion of the particles is

$$
m \frac{d^{2} \Delta \vec{x}}{d t^{2}}=\vec{f}_{\text {light }}(\Delta \vec{x})-b \frac{d \Delta \vec{x}}{d t},
$$

where $\vec{f}_{\text {light }}$ is the optical force and $b$ is the damping constant. The frictional term in (B2) is added to account for the friction between the particles and the environment that is often present in reality. If the displacement (B1) is small, it is possible to simplify (B2) with a linear approximation with respect to the displacement, the resultant linearized equation of motion is

$$
m \frac{d^{2} \Delta \vec{x}}{d t^{2}} \approx \vec{f}_{0}+\overleftrightarrow{K} \Delta \vec{x}-b \frac{d \Delta \vec{x}}{d t}
$$

where

$$
\vec{f}_{0}=\left(F_{0 x}, F_{0 y}, F_{0 x}, F_{0 y}, \ldots, F_{0 x}, F_{0 y}\right)
$$

is the drifting force that drives the whole cluster to move in unison and is equal to zero for static equilibrium, $m$ is the sphere's mass, and

$$
(\overleftrightarrow{K})_{j k}=\frac{\partial\left(\vec{f}_{\text {light }}\right)_{j}}{\partial \Delta x_{k}}
$$

is the force constant matrix.

To solve (B3), it is more appropriate to work with a coordinate system that is comoving with the whole cluster

$$
\Delta x^{\prime}(t)= \begin{cases}\Delta x(t) & \text { if } \vec{f}_{0}=0, \\ \Delta x(t)-\vec{f}_{0} t / b & \text { if } \vec{f}_{0} \neq 0 \text { and } b \neq 0, \\ \Delta x(t)-\vec{f}_{0} t^{2} / 2 m & \text { if } \vec{f}_{0} \neq 0 \text { and } b=0 .\end{cases}
$$

The $\vec{f}_{0}$ term in (B3) is now eliminated and the linearized equation of motion (B3) becomes

$$
m \frac{d^{2} \Delta \vec{x}^{\prime}}{d t^{2}} \approx \stackrel{\leftrightarrow}{K} \Delta \vec{x}^{\prime}-b \frac{d \Delta \vec{x}^{\prime}}{d t} .
$$

Introducing the transformation

$$
\Delta \vec{x}^{\prime}=\stackrel{\leftrightarrow}{V} \vec{\eta}
$$

where the columns of $\overleftrightarrow{V}$ are the eigenvectors of $\overleftrightarrow{K}$ so that $\overleftrightarrow{K}$ is diagonalized with eigenvalues $K_{i}$ 's

$$
\stackrel{\leftrightarrow}{V^{-1}} \stackrel{\leftrightarrow}{K} \stackrel{\leftrightarrow}{V}=\sum_{i} \hat{x}_{i} \hat{x}_{i}^{T} K_{i}
$$

Note that as the force is nonconservative (see main text), the force constant matrix $\overleftrightarrow{K}$ is a real-valued nonsymmetrical matrix. As a result, the eigenvalues of $\overleftrightarrow{K}$ and thus their corresponding eigenvectors, can be real numbers or conjugate pairs of complex numbers. After substituting (B8) into (B7), the equation of motion is now decoupled and reads

$$
m \frac{d^{2} \eta_{i}}{d t^{2}}=K_{i} \eta_{i}-b \frac{d \eta_{i}}{d t} .
$$

Equation (B10) is a second order linear ordinary differential equation which may be solved by the standard technique of substituting

$$
\eta_{i}=\eta_{0 i} e^{i \Omega_{i} t},
$$

where $\eta_{0 i}$ is a constant independent of time. It turns out that the solution to (B10) can be categorized according to the eigenvalues of $K_{i}$ or equivalently the natural frequency of the eigenmode defined as

$$
\Omega_{i 0}=\sqrt{-K_{i} / m} .
$$

In the following, we consider different types of eigenmodes of the photonic clusters.

\section{Neutral mode characterized by a zero natural frequency}

As the intensity on the trapping plane is homogeneous, the optical force acting on the particles can only depend on the relative positions between the particles. Consequently every photonic cluster must have two neutral modes with $K_{i}$ $=0$, corresponding to uniform motion of the whole cluster within the trapping plane. The eigenmodes are

$$
\begin{aligned}
& \Delta \vec{x}_{\text {neutral }, x}^{\prime}(t)=(1,0,1,0, \ldots, 1,0)\left(A_{x}+B_{x} e^{-b t / m}\right), \\
& \Delta \vec{x}_{\text {neutral }, y}^{\prime}(t)=(0,1,0,1, \ldots, 0,1)\left(A_{y}+B_{y} e^{-b t / m}\right),
\end{aligned}
$$

where $\left\{A_{x}, B_{x}, A_{y}, B_{y}\right\}$ are constants to be determined from initial conditions.

\section{Unstable mode characterized by an imaginary natural frequency}

If $K_{i}$ is real and positive, the corresponding natural frequency is purely imaginary and the eigenmode is

$$
\Delta \vec{x}_{i}^{\prime}(t)=e^{-b t / 2 m} \vec{V}_{i}\left[A_{i} e^{-t \sqrt{(b / 2 m)^{2}+\left|\Omega_{i 0}\right|^{2}}}+B_{i} e^{t \sqrt{(b / 2 m)^{2}+\left|\Omega_{i 0}\right|^{2}}}\right]
$$

where $A_{i}$ and $B_{i}$ are constants to be determined from the initial conditions. The mode is unstable because (B15) diverges with time.

\section{Stable mode characterized by real natural frequency}

If $K_{i}$ is real and negative, the natural frequency is purely real and the motions of the particles are that of a damped harmonic oscillator. 


$$
\begin{aligned}
& \text { For }(b / 2 m)^{2}>\Omega_{i 0}^{2}, \\
& \qquad \Delta \vec{x}_{i}^{\prime}(t)=e^{-b t / 2 m} \vec{V}_{i}\left[A_{i} e^{-t \sqrt{(b / 2 m)^{2}-\Omega_{i 0}^{2}}}+B_{i} e^{t \sqrt{(b / 2 m)^{2}-\Omega_{i 0}^{2}}}\right],
\end{aligned}
$$

where $A_{i}$ and $B_{i}$ are constants to be determined from initial conditions. The oscillation is overdamped.

For $(b / 2 m)^{2}=\Omega_{i 0}^{2}$,

$$
\Delta \vec{x}_{i}^{\prime}(t)=e^{-b t / 2 m} \vec{V}_{i}\left[A_{i}+B_{i} t\right],
$$

where $A_{i}$ and $B_{i}$ are constants to be determined from initial conditions. The oscillation is critically damped.

For $(b / 2 m)^{2}<\Omega_{i 0}^{2}$,

$$
\Delta \vec{x}_{i}^{\prime}(t)=A_{i} e^{-b t / 2 m} \vec{V}_{i} \sin \left[\sqrt{\Omega_{i 0}^{2}-(b / 2 m)^{2}} t+\phi_{i}\right]
$$

where $A_{i}$ and $\phi_{i}$ are constants to be determined from initial conditions. The oscillation is underdamped.

Eigenmodes (B16)-(B18) all converge to the origin as time increases, thus they are all stable.

\section{Complex mode characterized by a complex natural frequency}

In conservative mechanical system, the eigenvalues of the force constant matrix is always real valued, because the force constant matrix is symmetrical. However, it is not the case for the light bounded photonic cluster where conjugate pairs of complex valued eigenvalues exist. To obtain the pair of eigenmodes associated with the conjugate pair of eigenvalues $K_{i}$ and $K_{i+1}=K_{i}^{*}$, it suffices to consider only $K_{i}$ where $\operatorname{Im}\left(K_{i}\right)>0$. It turns out that the solutions associated with $K_{i+1}$ are linearly dependent with that of $K_{i}$. The eigenmodes are

$$
\begin{gathered}
\Delta \vec{x}_{i+}^{\prime}(t)=a_{i} e^{-\operatorname{Im}\left(\Omega_{i+}\right) t}\left\{\begin{array}{l}
\operatorname{Re}\left(\vec{V}_{i}\right) \sin \left[\operatorname{Re}\left(\Omega_{i+}\right) t+\phi_{i a}\right] \\
+\operatorname{Im}\left(\vec{V}_{i}\right) \cos \left[\operatorname{Re}\left(\Omega_{i+}\right) t+\phi_{i a}\right]
\end{array}\right\}, \\
\Delta \vec{x}_{i-}^{\prime}=b_{i} e^{-\operatorname{Im}\left(\Omega_{i-}\right) t}\left\{\begin{array}{l}
\operatorname{Re}\left(\vec{V}_{i}\right) \sin \left[\operatorname{Re}\left(\Omega_{i-}\right) t+\phi_{i b}\right] \\
+\operatorname{Im}\left(\vec{V}_{i}\right) \cos \left[\operatorname{Re}\left(\Omega_{i-}\right) t+\phi_{i b}\right]
\end{array}\right\},
\end{gathered}
$$

where $\left\{a_{i}, b_{i}, \phi_{i a}, \phi_{i b}\right\}$ are constants to be determined from initial conditions,

$$
\operatorname{Re}\left(\Omega_{i \pm}\right)=\frac{\mp\left[\left(b^{2}+4 m \operatorname{Re}\left\{K_{i}\right\}\right)^{2}+16 m^{2} \operatorname{Im}\left\{K_{i}\right\}^{2}\right]^{1 / 4} \sin \left(\delta_{i} / 2\right)}{2 m},
$$

$\operatorname{Im}\left(\Omega_{i \pm}\right)=\frac{b \pm\left[\left(b^{2}+4 m \operatorname{Re}\left\{K_{i}\right\}\right)^{2}+16 m^{2} \operatorname{Im}\left\{K_{i}\right\}^{2}\right]^{1 / 4} \cos \left(\delta_{i} / 2\right)}{2 m}$

and

$$
\delta_{i}= \begin{cases}\tan ^{-1} \frac{4 m\left|\operatorname{Im}\left\{K_{i}\right\}\right|}{b^{2}+4 m \operatorname{Re}\left\{K_{i}\right\}} & \text { if } b^{2}+4 m \operatorname{Re}\left\{K_{i}\right\}>0, \\ \pi-\tan ^{-1} \frac{4 m\left|\operatorname{Im}\left\{K_{i}\right\}\right|}{\left|b^{2}+4 m \operatorname{Re}\left\{K_{i}\right\}\right|} & \text { if } b^{2}+4 m \operatorname{Re}\left\{K_{i}\right\}<0 .\end{cases}
$$

\section{a. Complex unstable mode}

If $\operatorname{Re}\left(K_{i}\right)>0$, the $\Delta \vec{x}_{i+}^{\prime}(t)$ mode is spiraling inward to the equilibrium thus stable, whereas the $\Delta \vec{x}_{i-}^{\prime}(t)$ mode is spiraling outward and the displacement diverges with time, thus unstable. Consequently, a photonic cluster having a complex $K_{i}$ with positive real part is unstable and we denote this kind of eigenmode as complex unstable mode.

\section{b. Quasistable mode}

If $\operatorname{Re}\left(K_{i}\right)<0$, the $\Delta \vec{x}_{i+}^{\prime}(t)$ mode is spiraling inward to the equilibrium thus stable. The $\Delta \vec{x}_{i-}^{\prime}(t)$ mode requires some attention. The mode is spiraling outward if

$$
b<b_{\text {critical }}=\frac{\sqrt{m}\left|\operatorname{Im}\left(K_{i}\right)\right|}{\sqrt{\left|\operatorname{Re}\left(K_{i}\right)\right|}},
$$

but spiraling inward if

$$
b>b_{\text {critical }} \text {. }
$$

We denote this kind of eigenmode as quasistable, where the stability depends on the damping provided by the environment. The point $b=b_{\text {critical }}$ is hyperbolic, as already discussed in the text, linear stability analysis was not sufficient to determine the stability. Molecular dynamics simulations were performed and the results are plotted on Figs. 6-9.

The general solution to the linearized equation of motion (B3) is a linear combination of the neutral mode, unstable mode, stable mode, complex unstable mode, and quasistable mode according to the analysis above. If the eigenmodes of a photonic cluster consist of only neutral mode(s) and stable mode(s), it is called stable. If an unstable mode or complex unstable is present, it is called unstable. If the eigenmodes of a photonic cluster consists of neutral mode(s), stable mode(s), and quasistable mode(s), it is called quasistable. For quasistable photonic clusters, molecular dynamics reveal that the cluster may lock into a periodic orbit if appropriate friction is provided.

\footnotetext{
*Present address: Department of Physics, Fudan University, Shanghai, China.

$\dagger$ Author to whom correspondence should be addressed. Email address: phchan@ust.hk
}

${ }^{1}$ C. F. Bohren and D. R. Huffman, Absorption and Scattering of Light by Small Particles (Wiley, New York, 1983).

${ }^{2}$ S. Chu, J. E. Bjorkholm, A. Ashkin, and A. Cable, Phys. Rev. Lett. 57, 314 (1986). 
${ }^{3}$ A.Ashkin, J. M. Dziedzic, J. E. Bjorkholm, and S. Chu, Opt. Lett. 11, 288 (1986).

${ }^{4}$ A. Ashkin, IEEE J. Sel. Top. Quantum Electron. 6, 841 (2000).

${ }^{5}$ D. G. Grier, Nature (London) 424, 810 (2003).

${ }^{6}$ J. E. Molloy and M. J. Padgett, Contemp. Phys. 43, 241 (2002).

${ }^{7}$ A. Ashkin and J. M. Dziedzic, Science 235, 1517 (1987).

${ }^{8}$ K. Svoboda, P. P. Mitra, and S. M. Block, Proc. Natl. Acad. Sci. U.S.A. 91, 11782 (1994).

${ }^{9}$ C. Bustamante, S. B. Smith, J. Liphardt, and D. Smith, Curr. Opin. Struct. Biol. 10, 279 (2000).

${ }^{10}$ W. Hu, H. Li, B. Cheng, J. Yang, Z. Li, J. Xu, and D. Zhang, Opt. Lett. 20, 964 (1995).

${ }^{11}$ M. P. MacDonald, G. C. Spalding, and K. Dholakia, Nature (London) 426, 421 (2003).

${ }^{12}$ A. H. Chowdhury, B. J. Ackerson, and N. A. Clark, Phys. Rev. Lett. 55, 833 (1985).

${ }^{13}$ M. M. Burns, J. M. Fournier, and J. A. Golovchenko, Phys. Rev. Lett. 63, 1233 (1989).

${ }^{14}$ M. M. Burns, J. M. Fournier, and J. A. Golovchenko, Science 249, 749 (1990).

${ }^{15}$ J.-M. Fournier, M. M. Burns, and J. A. Golovchenko, Proc. SPIE 2406, 101 (1995).

${ }^{16}$ P. C. Chaumet and M. Nieto-Vesperinas, Phys. Rev. B 64, 035422 (2001).

${ }^{17}$ F. Claro and R. Rojas, Appl. Phys. Lett. 65, 2743 (1994).

${ }^{18}$ R. Fuchs and F. Claro (unpublished).

${ }^{19}$ S. Chu, Rev. Mod. Phys. 70, 685 (1998).

${ }^{20}$ C. E. Wieman, D. E. Pritchard, and D. J. Wineland, Rev. Mod. Phys. 71, S253 (1999).

${ }^{21}$ P. Domokos and H. Ritsch, Phys. Rev. Lett. 89, 253003 (2002).

${ }^{22}$ H. W. Chan, A. T. Black, and V. Vuletic, Phys. Rev. Lett. 90, 063003 (2003).

${ }^{23}$ A. T. Black, H. W. Chan, and V. Vuletic, Phys. Rev. Lett. 91, 203001 (2003).

${ }^{24}$ V. Garces-Chavez, K. Dholakia, and G. C. Spalding, Appl. Phys. Lett. 86, 031106 (2005).

25 It was Burns et al. who reported the quantitative measurement of the optical binding force. We also note that the interparticle, light-induced forces were observed by A. Ashkin in optical levi- tation of liquid drops in the 1970s, see A. Ashkin and J. M. Dziedzic, Science 187, 1073 (1975).

${ }^{26}$ S. A. Tatarkova, A. E. Carruthers, and K. Dholakia, Phys. Rev. Lett. 89, 283901 (2002).

${ }^{27}$ W. Singer, M. Frick, S. Bernet, and M. Ritsch-Marte, J. Opt. Soc. Am. B 20, 1568 (2003).

${ }^{28}$ X. Zhu, Y. Ling, G. Huang, H. Zhou, Y. Dai, K. Wu, and Z. Gan, Chin. Phys. Lett. 15, 165 (1998).

${ }^{29}$ H. Eckstein and U. Kreibig, Z. Phys. D: At., Mol. Clusters 26, 239 (1993).

${ }^{30}$ F. Depasse and J.-M. Vigoureux, J. Phys. D 27, 914 (1994).

${ }^{31}$ D. McGloin, A. E. Carruthers, K. Dholakia, and E. M. Wright, Phys. Rev. E 69, 021403 (2004).

${ }^{32}$ J. D. Jackson, Classical Electrodynamics, 3rd ed., (Wiley, New York, 1999), p. 261.

${ }^{33}$ Y. L. Xu, Appl. Opt. 34, 4573 (1995).

${ }^{34}$ M. H. Gutknecht, SIAM J. Sci. Comput. (USA) 12, 1020 (1993).

${ }^{35}$ For a discussion on different algorithm for electromagnetic scattering, see M. I. Mishchenko, L. D. Travis, and A. A. Lacis, Scattering, Absorption, and Emission of Light by Small Particles (Cambridge University Press, Cambridge, 2002), Chap. 6.

${ }^{36}$ J. Israelachvili, Intermolecular and Surface Forces, 2nd ed. (Academic Press, London, 1991).

${ }^{37}$ P. C. Chaumet and M. Nieto-Vesperinas, Opt. Lett. 25, 1065 (2000).

${ }^{38}$ M. I. Antonoyiannakis and J. B. Pendry, Europhys. Lett. 40, 613 (1997).

${ }^{39}$ J. Ng, Z. F. Lin, C. T. Chan, and P. Sheng, Europhys. Lett. 30 (15), 1956 (2005).

${ }^{40}$ See, e.g., P. Glendinning, Stability, Instability and Chaos (Cambridge University Press, Cambridge 1994), Chap. 7.

${ }^{41}$ S. Stein, Q. Appl. Math. 19, 15 (1961).

${ }^{42}$ O. R. Cruzan, Q. Appl. Math. 20, 33 (1962).

${ }^{43}$ Y. L. Xu, J. Comput. Phys. 139, 137 (1998).

${ }^{44}$ M. I. Mishchenko, Light Scattering by Nonspherical Particles (Academic, New York, 2000), pp. 267-271. 